\title{
Two Many Values: An algorithmic outlook on Suszko's Thesis
}

\author{
Carlos Caleiro \\ SQIG-Instituto de Telecomunicações, and \\ Dept. Mathematics, IST, TU-Lisbon \\ Email: ccal@math.ist.utl.pt
}

\author{
João Marcos \\ LoLITA and DIMAp, UFRN, and \\ Institut für Computersprachen E1852, TU-Wien \\ Email: jmarcos@dimap.ufrn.br
}

\begin{abstract}
In spite of the multiplication of truth-values, a noticeable shade of bivalence lurks behind the canonical notion of entailment that many-valued logics inherit from the 2 -valued case. Can this bivalence be somehow used to our advantage? The present note briefly surveys the progress made in the last three decades toward making that theme precise from an abstract point of view and extracting some useful procedures from it, harvesting some of its most favorable crops on the domains of semantics and proof-theory.
\end{abstract}

\section{ANTIDOTES For 'A MAGNIFICENT CONCEPTUAL DECEIPT'}

In a 1976 lecture (cf. [39]), the Polish logician Roman Suszko complained that "after 50 years [of the construction of so-called many-valued logics by Jan Łukasiewicz] we still face an illogical paradise of many truths and falsehoods". The bold philosophical thesis behind such an assertion (cf. [37]), updating and extending Frege's discrimination between the sense and the reference of saturated concepts, was that a sharp distinction should be drawn in between the 'algebraic valuations' of the most usual multi-valued truth-functional logics, and their more 'genuine definition' in terms of twovalued 'logical valuations' (cf. [38]).

Suszko's Thesis, as formulated in [26] and [20], roughly says that "every logic is logically two-valued". To put it like that, however, would result in allowing for circumstances in which it is outright wrong, others in which it is but trivial, and still some others in which it is just useless. To do the Thesis some justice, show how and when it works fine, and to find some nice applications for it, we will need some preparation, to be supplied in the present section.

As usual in the general theory of consequence relations (cf. [41]), a propositional logic $\mathcal{L}$ will here be characterized as a collection of formulas $\mathcal{S}$ together with a singleconclusion consequence relation somehow defined as a subset of $\operatorname{Pow}(\mathcal{S}) \times \mathcal{S}$. Moreover, following Łoś \& Suszko's methodological work on sentential logics (cf. [24]), we will assume $\mathcal{S}$ to be freely generated by a denumerable set of atoms At $=$ $\left\{p_{0}, p_{1}, p_{2}, \ldots\right\}$ over the constructors Cct $=\bigcup_{m \in \mathbb{N}} \mathrm{Cct}_{m}$, where each $\mathrm{Cct}_{m}$ itself denotes a collection of connectives of arity $m$. We will call a set of formulas $\Sigma$ overcomplete in $\mathcal{L}=\langle\mathcal{S}, \Vdash\rangle$ in case $\Sigma \Vdash \beta$ for every $\beta \in \mathcal{S}$. Taking advantage of the algebraic character of $\mathcal{S}$, for any given total substitution mapping $\sigma:$ At $\rightarrow \mathcal{S}$ there will of course be a unique endomorphism $\varepsilon^{\sigma}: \mathcal{S} \rightarrow \mathcal{S}$ that extends it, and we will assume henceforth that the consequence relation $\Vdash$ of each of our logics enjoys the following property of substitutionality (a.k.a. 'structurality'):

$$
\text { (L0) } \quad \Gamma \Vdash \alpha \text { implies } \varepsilon^{\sigma}(\Gamma) \Vdash \varepsilon^{\sigma}(\alpha)
$$

It will help in the following to denote by $\operatorname{At}(\Sigma)$ the set of atoms that occur in the construction of a given theory $\Sigma \subseteq \mathcal{S}$.

From a semantical point of view, let an interpretation for the formulas in $\mathcal{S}$ be a total valuation mapping $\S: \mathcal{S} \rightarrow \mathcal{V}_{\S}$ into a given universe of truth-values $\mathcal{V}_{\S}$, and consider $\mathcal{V}_{\S}$ to be partitioned into sets of designated values $\mathcal{D}_{\S}$ and undesignated values $\mathcal{U}_{\S}$. A many-valued semantics Sem here will be any collection of such valuations. From these elements, local $\left(\models_{\S}\right)$ and global $(\models \mathrm{Sem})$ consequence relations may then be defined according to a canonical concept of $T$-entailment that sets $\Gamma \models_{\S} \alpha$ iff $(\S(\gamma) \in \mathcal{U}$, for some $\gamma \in \Gamma$, or $\S(\alpha) \in \mathcal{D})$, and sets $\Gamma \models_{\text {Sem }} \alpha$ iff $\left(\Gamma=_{\S} \alpha\right.$, for every $\S \in$ Sem).

It has since long been known that a consequence relation $\Vdash$ over $\mathcal{S}$ can be characterized by an adequate $T$-entailment relation $\models_{\text {Sem }}$ iff it enjoys the following properties, for arbitrary $\Gamma \cup \Delta \cup\{\alpha\} \subseteq \mathcal{S}:$

(L1) $\Gamma, \alpha \Vdash \alpha$

(L2) $\quad \Gamma \Vdash \alpha$ implies $\Delta \cup \Gamma \Vdash \alpha$

(L3) $\quad(\Gamma \Vdash \delta$, for every $\delta \in \Delta$, and $\Delta \Vdash \alpha)$ imply $\Gamma \Vdash \alpha$

Let's call this result W-theorem (cf. [41]). Consider now the set of 'logical' values $\mathcal{V}_{2}=\{T, F\}$ such that $\mathcal{D}_{2}=\{T\}$, and for each mapping $\S \in \mathcal{S}$ let its bivalent counterpart $b_{\S}$ : $\mathcal{S} \rightarrow \mathcal{V}_{2}$ be defined by setting $b_{\S}(\varphi)=T$ iff $\S(\varphi) \in \mathcal{D}$. Collecting all such bivalent mappings into $\mathrm{Sem}_{2}$, it is obvious that $\Gamma=_{\mathrm{Sem}} \alpha$ iff $\Gamma=_{\mathrm{Sem}_{2}} \alpha$. This may be said to constitute indeed the very core of Suszko's observation on logical 2valuedness, and we will call this result S-theorem. A quick review of the above mentioned theorems and their proofs, also extended to the multiple-conclusion case, can be found in [28].

A particularly interesting genre of many-valued semantics Sem is obtained when one fixes the sets $\mathcal{V}_{\S}$ and $\mathcal{D}_{\S}$ (call them $\mathcal{V}$ and $\mathcal{D}$ ), for every $\S \in$ Sem, and also fixes the interpretation $\lceil$ (c) $\rceil$ of each (c) $\in \mathrm{Cct}_{m}$ in such a way that, for every $\S \in$ Sem and formulas $\alpha_{0}, \ldots, \alpha_{m-1}$, the following equation holds good:

$$
\S\left(\text { (c) }\left(\alpha_{0}, \ldots, \alpha_{m-1}\right)\right)=\lceil(\rceil\rceil\left(\S\left(\alpha_{0}\right), \ldots, \S\left(\alpha_{m-1}\right)\right)
$$


This means that we may think now of the universe of truthvalues $\mathcal{V}$ as organized in terms of an algebra with the same similarity type of the algebra of formulas, where to each syntactical constructor (c) : $\mathcal{S}^{m} \rightarrow \mathcal{S}$ there corresponds a semantical operator $\lceil(\mathrm{C})\rceil: \mathcal{V}^{m} \rightarrow \mathcal{V}$. This also means, of course, that any basic state of affairs given by a total mapping $e:$ At $\rightarrow \mathcal{V}$ can be uniquely extended into a homomorphic valuation $\S^{e}: \mathcal{S} \rightarrow \mathcal{V}$ from the algebra of formulas into the algebra of truth-values. Any semantics given by the collection of all such homomorphisms is called truth-functional. Now, say that the sets of formulas $\Sigma$ and $\Pi$ are disconnected in case $\operatorname{At}(\Sigma) \cap \operatorname{At}(\Pi)=\varnothing$. A remarkable result by Shoesmith $\&$ Smiley (cf. [35]) shows that a logic is characterized by a truthfunctional $T$-entailment iff it enjoys all the (L\#)-properties above, plus the following cancellation property:

(L4) $\bigcup_{k \in K} \Gamma_{k} \cup \Gamma \Vdash \varphi$ implies $\Gamma \Vdash \varphi$, once, for every $k \in K$, we have that $\Gamma \cup\{\varphi\}$ and $\Gamma_{k}$ are disconnected, and that $\Gamma_{k}$ is not overcomplete in this logic

A $\operatorname{logic} \mathcal{L}$ is said to be genuinely $\kappa$-valued if $\kappa$ is the cardinality of the smallest collection of truth-values $\mathcal{V}_{\kappa}$ with the help of which $\mathcal{L}$ can be given a truth-functional semantics. The drama set up by the S-theorem reaches its climax exactly in the cases in which $\mathcal{L}$ turns out to be genuinely $\kappa$-valued, for some $\kappa>2$ : here a bivalent characterization of $\mathcal{L}$ will presume an open abandonment of a truth-functional characterization.

A genuinely $\kappa$-valued logic $\mathcal{L}$ with a set of constructors Cct is said to be functionally complete in case any operator $\lceil\circledast\rceil$ over $\mathcal{V}_{\kappa}$ can be defined by way of some convenient combination of operators associated to the constructors Cct. Consider any two distinct values $v_{i}, v_{j} \in \mathcal{V}_{\kappa}$, let $\theta^{i j}$ be such that $\operatorname{At}\left(\left\{\theta^{i j}\right\}\right)=\left\{p_{0}\right\}$ and let $\sigma_{\left[p_{k} \mapsto \delta\right]}$ be a substitution mapping that outputs the value $\delta$ with input $p_{k}$ and behaves as the identity mapping otherwise. Given a state of affairs $e$ such that $e\left(p_{i}\right)=v_{i}$ and $e\left(p_{j}\right)=v_{j}$, and its corresponding valuation $\S^{e}$, we say that the formula $\theta^{i j}$ effectively separates $v_{i}$ and $v_{j}$ in case $b_{\S e}\left(\varepsilon_{\left[p_{0} \mapsto p_{i}\right]}^{\sigma}\left(\theta^{i j}\right)\right) \neq b_{\S}\left(\varepsilon_{\left[p_{0} \mapsto p_{j}\right]}^{\sigma}\left(\theta^{i j}\right)\right)$. Obviously, it suffices to take $\theta^{i j}$ as $p_{0}$ itself to separate truthvalues that are not both designated, nor both undesignated. For pairs of values from the same partition class, however, it may or it may not be the case that the logic $\mathcal{L}$ has the linguistic resources to separate them. We will here say that a genuinely $\kappa$-valued logic $\mathcal{L}$ is sufficiently expressive when its language is expressive enough to separate each pair of truth-values from the collection $\mathcal{V}_{\kappa}$. Clearly, functional completeness gives a sufficient condition, yet, as we shall see, not a necessary one, for a logic to be sufficiently expressive. Notice moreover that, as we have proven in [15], for any genuinely $\kappa$-valued logic $\mathcal{L}$, with $\kappa>2$, either $\mathcal{L}$ or some conservative extension of it (not necessarily a truth-functional one) is bound to be sufficiently expressive.

Back from semantics to abstract properties of consequence relations, given a logic $\mathcal{L}=\langle\mathcal{S}, \Vdash\rangle$, we say that two formulas $\gamma$ and $\delta$ are $\mathcal{L}$-equivalent, and denote this by $\gamma \equiv_{\mathcal{L}} \delta$, if both $\{\gamma\} \Vdash \delta$ and $\{\delta\} \Vdash \gamma$. An important feature of classical logic, shared also by all the usual modal logics, is given by the enjoyment of the so-called replacement property, according to which equivalent formulas are 'logically indistinguishable', that is:

(L5) $\alpha \equiv_{\mathcal{L}} \beta$ implies $\varepsilon_{[q \mapsto \alpha]}(\varphi) \equiv_{\mathcal{L}} \varepsilon_{[q \mapsto \beta]}(\varphi)$, for any $\varphi \in \mathcal{S}$ and any $q \in$ At

Suszko sometimes called this property 'Fregean Axiom' (cf. [37], [39]) and claimed that "the construction of [the] so-called many-valued logics by Jan Łukasiewicz was the effective abolition of the Fregean Axiom". However, it must be highlighted that the claim in that case is only true, in fact, for sufficiently expressive logics. There are indeed genuinely $\kappa$-valued logics, with $\kappa>2$, that enjoy the replacement property: a simple example would be that of a truth-functional logic $\mathcal{L}^{\circledast}$ with $\mathcal{V}=\left\{v_{0}, v_{1}, v_{2}\right\}, \mathcal{D}=\left\{v_{2}\right\}$, and a single binary constructor $\circledast$ interpreted by setting $\lceil\circledast\rceil\left(v_{k}, v_{k}\right)=v_{k}$, for $k \in\{0,1,2\}$, and $\lceil\circledast\rceil\left(v_{i}, v_{j}\right)=v_{2}$, otherwise. A corrected version of Suszko's claim should then be something like: "a sufficiently expressive truth-functional logic may only satisfy the replacement property in case it is genuinely 2 -valued". A sufficiently expressive conservative extension of $\mathcal{L}^{\circledast}$ could be obtained for instance by adding to the language of this logic a 0 -ary constructor $\otimes$ interpreted by setting $[\nabla\rceil=v_{0}$, but then of course this logic would fail the replacement property (notice how (L5) fails if one considers, e.g., $\alpha=p_{0}, \beta=p_{0} \circledast \bigotimes$ and $\left.\varphi=q \circledast p_{0}\right)$. Once we will be interested below exclusively on sufficiently expressive many-valued logics, all the nonclassical truth-functional logics we will consider are indeed to fail replacement - and this fact would certainly gratify Suszko in his analysis of the Fregean Axiom.

Several other important aspects of truth-functionality are discussed in [31], where open problems related to 'computationally well-behaved' generalizations of this very notion of truth-functionality are also mentioned. An interesting nondeterministic variety of truth-functionality has been proposed, for instance, in [2], where again the sets of truth-values are fixed for all interpretation mappings, but now for each (c) $\in \mathrm{Cct}_{m}$ there is an operator $\lfloor$ (c) $\rfloor: \mathcal{V}^{m} \rightarrow \operatorname{Pow}(\mathcal{V}) \backslash \varnothing$ such that, for every $\S \in$ Sem and formulas $\alpha_{0}, \ldots, \alpha_{m-1}$ :

(S2) $\S\left(\right.$ C $\left.\left(\alpha_{0}, \ldots, \alpha_{m-1}\right)\right) \in\left\lfloor(\mathcal{C}\rfloor\left(\S\left(\alpha_{0}\right), \ldots, \S\left(\alpha_{m-1}\right)\right)\right.$

This means that there might be a number of ways of interpreting the meaning of each constructor as applied to a given tuple of inputs. Consider for instance the simple example of a logic with having a binary constructor $\supset$ interpreted deterministically over $\mathcal{V}_{2}=\{T, F\}, \mathcal{D}_{2}=\{T\}$, and $\mathcal{U}_{2}=\{F\}$ as the classical implication, that is, such that $v_{1}\lfloor\supset\rfloor v_{2} \in \mathcal{U}_{2}$ iff $\left(v_{1} \in \mathcal{D}_{2}\right.$ and $\left.v_{2} \in \mathcal{U}_{2}\right)$, and having a 0 -ary constructor I interpreted non-deterministically by setting $\mid I\rfloor=\mathcal{V}_{2}$. In that case the resulting logic would not enjoy property (L4) (just consider $K=\{0\}, \Gamma_{0}=\left\{p_{0} \supset \mathrm{I}, p_{0}\right\}, \Gamma=\varnothing$ and $\left.\varphi=p_{1} \supset \mathrm{I}\right)$, and would fail thus to be truth-functional (cf. [31]). It is not entirely clear, however, what the meaning of Suszko's Thesis on logical two-valuedness would be in such a scenario, and in particular it is not as yet known how the class of consequence relations related to such a wider class of non- 
deterministically truth-functional logics is to be characterized from an abstract viewpoint.

The next sections will show how logical two-valuedness have been explored from a constructive and implementationoriented perspective. To be perfectly fair, however, we will end the present section by briefly mentioning some ways in which a logic may fail to be bivalent, even in the sense of the S-theorem. The obvious way of obtaining that effect, of course, would be by proposing consequence relations that fail some of the (L\#)-properties. Such is the case of the notion of 'inferential many-valuedness' studied in [27], that goes against Suszko's Thesis in that it turns out to be based on 'logical 3-valuedness' and a slightly modified notion of entailment. Another illuminating way of eluding the bivalence behind the notion of $T$-entailment would be by allowing either $\mathcal{V} \backslash(\mathcal{D} \cup \mathcal{U})$ or $\mathcal{D} \cap \mathcal{U}$ to be non-empty, as proposed in [40].

\section{THE EXTRACTION OF BIVALENT SEMANTICS FOR FINITE-VALUED LOGICS}

The employment of bivalent non-truth-functional semantics has proven extremely useful in the domain of non-classical logics, and especially when no other insightful varieties of semantics are available for those logics, at the time. The realms of paraconsistent and paracomplete logics have indeed benefitted a lot from the bivalent approach (cf. [23]), in particular in the case we are dealing with logics that both fail the replacement property and also fail to have genuinely $\kappa$ valued semantics, for finite $\kappa$ (cf. [18]). The pre-requisites for obtaining completeness for such bivalent semantics are now well understood (cf. [6]), and associated decidability procedures known as 'quasi matrices' have been used since [19]. Such procedures are in fact available, as we have argued in [8], at least when the clauses characterizing the bivalent semantics are presented in a certain specific 'dyadic' format.

Suszko's Thesis, however, is equally valid when the logics do have a finite-valued truth-functional semantics, and the next section will discuss the worthiness of the Thesis in such a domain. Before that, we will conclude the present section by succinctly appraising the more recent efforts toward constructively securing, prêt-à-porter, the finest consequences of the S-theorem.

One of the first announcements concerning the availability of a bivalent semantics for a genuinely 3-valued logic, Łukasiewicz's logic $Ł_{3}$, can be found in [38] — though the corresponding clauses concerning the collection of bivalent interpretation mappings appear only in [25]. One cannot exaggerate in asserting, however, that that specific adequate bivalent characterization for $\iota_{3}$, however, looked rather mystifying, as no effort was indeed made to clarify how it could be obtained directly from the set of truth-tables that characterize the original semantics of the logic. Given the considerably non-constructive character of the S-theorem, nonetheless, the definition of a constructive procedure for obtaining such a bivalent characterization should be particularly welcomed. A substantial step toward that goal was made in [5], where the author has as a matter of fact suggested that in many cases an 'algebraic' truth-value can be constructively exchanged by a unique 'binary print', in terms of a tuple of values from $\mathcal{V}_{2}$, with the exclusive help of the original linguistic resources of the given logic. In [8] we have explored a similar approach in order to constructively extract, from the specification of each given sufficiently expressive genuinely $\kappa$-valued logic, the clauses of a sound and complete bivalent semantics for it. The basic idea, to be sure, is to use the available linguistic resources to produce the effective separation of each pair of truth-values, and then use the corresponding syntactically expressed binary prints of those values to couch the original many-valued specification into a two-valued environment. A fuller study of how that procedure nicely realizes Suszko's Thesis and smoothly fits into the variegated many-valued scenarios from the literature was presented in [11] and [10].

\section{A BIRD'S EYE VIEW OF SOME APPLICATIONS TO MODEL THEORY AND TO PROOF THEORY}

A number of applications may be envisaged for bivalent semantics, some of which we will quickly examine in the present section. One of their most striking advantage, at first sight, lies in providing a uniform classic-like framework in which a plethora of different non-classical logics can be specified, and more easily compared with each other. From a model-theoretic viewpoint, besides helping in establishing decidability for a large class of non-classical logics, another productive application for a bivalent semantics consists in being a useful intermediary step in the process of associating another more informative kind of semantics for the same logic. Such has been the case, for instance, with the use of bivalent semantics in the proof of completeness of a certain semantics given by way of combinations of finite-valued truth-functional scenarios, even when the given non-classical logic turn out not to be characterizable by way of a genuinely finite-valued truth-functional semantics (cf. [29]). The underlying idea is somehow to 'split' a given complex logic in terms of more well-behaved ingredients (cf. [28]), a very generally applicable approach to model theory known as possible-translations semantics, first proposed in [16].

Now, for the case of logics that $d o$ have a finite-valued truthfunctional semantics, the constructive procedure for extracting a bivalent characterization for them, reported upon in the previous section, has borne some juicy fruits also from a prooftheoretical perspective. Even though general axiomatization algorithms for finite-valued logics have been known for long, they are typically based on indiscriminate extensions of the linguistic resources of the original logics, as in [36], or else they produce rules, as in [22] and [4], that do not easily allow for the comparison of a genuinely $\kappa_{1}$-valued to a genuinely $\kappa_{2}$-valued logic, when $\kappa_{1} \neq \kappa_{2}$. Such a general nonuniform approach to finite-valued logics in terms of tableaux, for example, has been available at least since [17]. Only recently, however, an algorithm has been proposed (cf. [8]) that produces tableau rules with only two labels, as in the classical case, exactly by exploring the underlying bivalence behind the notion of $T$-entailment, as supplied by our constructive 
rendering of the S-theorem. Furthermore, as argued in [32], the uniform classic-like approach may benefit even the user that wishes to compare the deductive strength of truth-functional logics based on the same structures, with $\kappa_{1}=\kappa_{2}$.

A full implementation of the above mentioned algorithm, receiving as input the specification of a sufficiently expressive finite-valued logic, together with the appropriate formulas that are able to produce the effective separation of each pair of truth-values, and producing as output a complete set of tableau rules as a ready-to-use Isabelle theory (cf. [34]) was presented in [33], and made available at http://tinyurl.com/5cakro. The tableau theory implemented in the framework of the higher-order metalanguage of a very flexible proof-assistant includes structural rules that allow for the relatively easy derivation, by the user, of theorems and rules of the given logics, as well as the comparison between different logics, all re-specified now in a uniform two-signed framework. Progress toward the complete automation of the associated proof procedures, however, was initially hindered by the fact that the set of tableau rules produced by the procedure laid out in [8] includes a kind of dual-cut branching rule that in principle would sanction the production of derivations that will not terminate, should the user make some bad choices along their construction. Though it had been known that in general this dual-cut rule was not eliminable, a conjecture had been made that all uses of cut in our systems could be made 'analytic', as in [21]. Such non-eliminable use of a clause representing the dual-cut rule was in fact an ordinary feature of the bivaluation semantics presented in dyadic format, as studied in [8], even for non-finite-valued logics.

Now, instead of proceeding toward directly proving the above mentioned conjecture about analytic cuts, for the finitevalued case, we have recently proposed, in [14], a novel algorithm that receives the very same many-valued specifications, and outputs adequate cut-free tableau systems. However, such systems allow for some non-determinism in the choice of rules for building the derivations, and in that case a bad choice of rule by the user in constructing his derivations could in fact result in non-termination. To fix that, and to guarantee deduction in the new systems to be completely automatic, our novel algorithm comes associated to a convenient proof strategy, in each case, based on a non-canonical measure of complexity of the involved formulas, to such an effect that the tableau system becomes 'analytic' in an extended sense, once the adherence to the proof strategy does guarantee termination of the task of verifying the validity of a given inference. Moreover, as usual, when that task terminates by producing a non-closed tableau, exhausted according to the new definition of complexity measure, full counter-models may be immediately extracted from the open branches of the tableau. Challenges and developments toward the implementation of this new axiom-extraction procedure, together with a fully automated deduction tactic in a computer-assisted environment, are reported in [30]. To extend the procedure from sufficiently expressive finite-valued truth-functional logics so as to be applicable to any finite-valued truth-functional logic, a conservative extension of the given input logic might of course be needed, as we have explained in Section I, and in [15] we show how such aim may be attained, introducing only 'minimal' changes to the original logic.

Analyticity has also been the focus of a recent study on non-deterministic semantics (cf. [1], and recall Section II), and this study has been showing some interesting counterparts in the modularity of the approach, though still not as much reflected in the development of uniform classic-like prooftheoretical frameworks. Canonical multi-signed sequent-style proof systems have been developed, at any rate, for this kind of semantics (cf. [3]). Cut-free classic-like sequent systems adequate for logics presented by way of a bivalent semantics have also been studied elsewhere (cf. [7]). In [9] we are to show how our novel classic-like automated axiomatization procedure may indeed be extended from bivalent semantics extracted from finite-valued logics to all other logics whose semantics can be specified in dyadic format, coupling the obtained proof system, in each case, with a convenient proof strategy originated from a non-canonical complexity measure.

Further extensions of such constructive procedures and strategies should target also genuinely infinite-valued logics, logics endowed with other kinds of semantics that generalize the traditional notion of truth-functionality, and first-order logics. Improvements on efficiency of the associated proof theories should be expected if the format of the extracted rules is modified, for instance, in order to have them be produced as KE-tableaux (cf. [21]), allowing for a finer negotiation with the notion of analyticity.

Suszko's Thesis is certainly fruitless if we regard it as a dogma, but it can be an insightful tool of logical analysis, as we hope to have illustrated here. Truth-functionality is for sure a nice and simple rule for our algebraic-oriented minds, but there is no reason to fear its absence, even from a strictly algebraic point of view, as results from recent developments in algebraic logic (cf. [13] and [12]) have shown.

\section{ACKNOWLEDGMENT}

The first author was partly supported by FCT and EU FEDER, namely via the KLog project PTDC/MAT/ 68723/2006 of SQIG-IT. The second author acknowledges partial support by CAPES.

\section{REFERENCES}

[1] A. Avron, "Multi-valued semantics: Why and how," Studia Logica, to appear.

[2] A. Avron and I. Lev, "Non-deterministic matrices," in Proceedings of the XXXIV International Symposium on Multiple-Valued Logic (ISMVL 2004). IEEE Computer Society, 2004, pp. 282-287.

[3] A. Avron and A. Zamansky, "Non-deterministic semantics for logical systems (a survey)," in Handbook of Philosophical Logic, 2nd ed., D. Gabbay and F. Guenthner, Eds. Kluwer, to appear.

[4] M. Baaz, C. G. Fermüller, and G. Salzer, "Automated deduction for many-valued logics," in Handbook of Automated Reasoning, J. A. Robinson and A. Voronkov, Eds. Elsevier and MIT Press, 2001, pp. 1355-1402.

[5] D. Batens, "A bridge between two-valued and many-valued semantic systems: $n$-tuple semantics," in Proceedings of the XII International Symposium on Multiple-Valued Logic. IEEE Computer Society, 1982, pp. 318-322. 
[6] J.-Y. Béziau, "Recherches sur la logique abstraite: Les logiques normales," Logika, vol. 18, pp. 105-114, 1998.

[7] —_ "Sequents and bivaluations," Logique et Analyse (N.S.), vol. 44, no. 176, pp. 373-394, 2001.

[8] C. Caleiro, W. Carnielli, M. E. Coniglio, and J. Marcos, "Two's company: "The humbug of many logical values"," in Logica Universalis, J.-Y. Béziau, Ed. Basel, Switzerland: Birkhäuser Verlag, 2005, pp. 169189, preprint available at: http://sqig.math.ist.utl.pt/pub/CaleiroC/05-CCCM-dyadic.pdf.

[9] C. Caleiro, W. A. Carnielli, M. E. Coniglio, and J. Marcos, "How many logical values are there? Dyadic semantics for many-valued logics," Draft, forthcoming.

[10] — "Dyadic semantics for many-valued logics," CLC, Department of Mathematics, Instituto Superior Técnico, 1049-001 Lisbon, PT, Research Report, 2003, presented at the III World Congress on Paraconsistency, Toulouse, FR, July 28-31, 2003. http://sqig.math.ist.utl.pt/pub/CaleiroC/03-CCCM-dyadic2.pdf.

[11] _ "Suszko's Thesis and dyadic semantics," CLC, Department of Mathematics, Instituto Superior Técnico, 1049-001 Lisbon, PT, Research Report, 2003, presented at the III World Congress on Paraconsistency, Toulouse, FR, July 28-31, 2003. http://sqig.math.ist.utl.pt/pub/CaleiroC/03-CCCM-dyadic1.pdf.

[12] C. Caleiro and R. Gonçalves, "Towards a behavioral algebraic theory of logical valuations," submitted for publication.

[13] — "Algebraic valuations as behavioral logical matrices," in Proceedings of the XVI Workshop on Logic, Language, Information and Computation (WoLLIC 2009), held in Tokyo, JP, June 2009, ser Lecture Notes in Artificial Intelligence, H. Ono, M. Kanazawa, and R. de Queiroz, Eds., vol. 5514. Springer, 2009, pp. 13-25, preprint available at: http://sqig.math.ist.utl.pt/pub/CaleiroC/09-CG-AlgValuations.pdf.

[14] C. Caleiro and J. Marcos, "Classic-like analytic tableaux for finitevalued logics," in Proceedings of the XVI Workshop on Logic, Language, Information and Computation (WoLLIC 2009), held in Tokyo, JP, June 2009, ser. Lecture Notes in Artificial Intelligence, H. Ono, M. Kanazawa, and R. de Queiroz, Eds., vol. 5514. Springer, 2009, pp. 268-280.

[15] — , "A uniform classic-like analytic deductive formalism for finitevalued logics," 2009, submitted to publication.

[16] W. Carnielli, "Many-valued logics and plausible reasoning," in Proceed ings of the XX International Symposium on Multiple-Valued Logic, held at the University of Charlotte / NC, US, 1990. IEEE Computer Society, 1990, pp. 328-335.

[17] W. A. Carnielli, "Systematization of the finite many-valued logics through the method of tableaux," The Journal of Symbolic Logic, vol. 52, no. 2, pp. 473-493, 1987.

[18] W. A. Carnielli, M. E. Coniglio, and J. Marcos, "Logics of Formal Inconsistency," in Handbook of Philosophical Logic, 2nd ed., D. Gabbay and F. Guenthner, Eds. Kluwer, 2007, vol. 14, pp. 1-93, preprint available at: http://sqig.math.ist.utl.pt/pub/MarcosJ/03-CCM-lfi.pdf.

[19] N. C. A. da Costa and E. Alves, "A semantical analysis of the calculi $C_{n}$, " Notre Dame Journal of Formal Logic, vol. 18, no. 4, pp. 621-630, 1977.

[20] N. C. A. da Costa, J.-Y. Béziau, and O. A. S. Bueno, "Malinowski and Suszko on many-valued logics: on the reduction of many-valuedness to two-valuedness," Modern Logic, vol. 3, pp. 272-299, 1996.

[21] M. D'Agostino and M. Mondadori, "The taming of the cut: classical refutations with analytic cut," Journal of Logic and Computation, vol. 4, no. 3, pp. 285-319, 1994.

[22] R. Hähnle, "Tableaux for many-valued logics," in Handbook of Tableau Methods, M. D'Agostino, D. Gabbay, R. Hähnle, and J. Posegga, Eds. Springer, 1999, pp. 529-580.
[23] A. Loparić and N. C. A. da Costa, "Paraconsistency, paracompleteness, and valuations," Logique et Analyse (N.S.), vol. 27, no. 106, pp. 119131,1984

[24] J. Łoś and R. Suszko, "Remarks on sentential logics," Indagationes Mathematicae, vol. 20, pp. 177-183, 1958.

[25] G. Malinowski, "Classical characterization of $n$-valued Łukasiewicz calculi," Reports on Mathematical Logic, vol. 9, pp. 41-45, 1977.

[26] G. Malinowski, Many-Valued Logics, ser. Oxford Logic Guides. Oxford: Clarendon Press, 1993, vol. 25.

[27] _ , "Inferential many-valuedness," in Philosophical Logic in Poland, J. Woleński, Ed. Dordrecht: Kluwer, 1994, pp. 75-84.

[28] J. Marcos, "Possible-translations semantics," in Proceedings of the Workshop on Combination of Logics: Theory and applications (CombLog'04), held in Lisbon, PT, July 28-30, 2004, W. A. Carnielli, F. M. Dionísio, and P. Mateus, Eds. Departamento de Matemática, Instituto Superior Técnico, 2004, pp. 119-128, extended version available at: http://sqig.math.ist.utl.pt/pub/MarcosJ/04-M-pts.pdf.

[29] _ - "Possible-translations semantics for some weak classically-based paraconsistent logics," Journal of Applied Non-Classical Logics, vol. 18, no. 1, pp. 7-28, 2008, preprint available at: http://sqig.math.ist.utl.pt/pub/MarcosJ/04-M-PTS4swcbPL.pdf.

[30] - "Automatic generation of proof tactics for finite-valued logics," 2009, to appear in Electronic Proceedings on Theoretical Computer Science. Preprint available at: http://sqig.math.ist.utl.pt/pub/MarcosJ/09-AGPT4FVL.pdf.

[31] _ "What is a non-truth-functional logic?" Studia Logica, vol. 92, pp. $215-240,2009$.

[32] _ "The value of the two values," LoLITA and DIMAp / UFRN, and Theory and Logic Group, TU-Wien, Extended abstract, 2010, presented at 31st Linz Seminar on Fuzzy Set Theory: Lattice-valued logic and its applications (LINZ 2010), Feb 9-13, 2009, Linz, AT.

[33] J. Marcos and D. Mendonça, "Towards fully automated axiom extraction for finite-valued logics," in The Many Sides of Logic, ser. Studies in Logic, W. Carnielli, M. E. Coniglio, and I. M. L. D’Ottaviano, Eds. London: College Publications, 2009, pp. 425-440, preprint available at: http://sqig.math.ist.utl.pt/pub/MarcosJ/08-MM-towards.pdf.

[34] T. Nipkow, L. C. Paulson, and M. Wenzel, Is abelle/HOL - A Proof Assistant for Higher-Order Logic, ser. LNCS. Springer, 2002, vol. 2283.

[35] D. J. Shoesmith and T. J. Smiley, "Deducibility and many-valuedness," The Journal of Symbolic Logic, vol. 36, no. 4, pp. 610-622, 1971.

[36] S. J. Surma, "An algorithm for axiomatizing every finite logic," in Computer Science and Multiple-Valued Logics, Selected Papers from the IV International Symposium on Multiple-Valued Logics, D. C. Rine, Ed. Amsterdam: North-Holland, 1974, pp. 143-149, 2nd edition, 1984.

[37] R. Suszko, "Abolition of the Fregean Axiom," in Logic Colloquium: Symposium on Logic held at Boston, 1972-73, ser. Lecture Notes in Mathematics, R. Parikh, Ed. Berlin: Springer-Verlag, 1972, vol. 453, pp. 169-239.

[38] — , "Remarks on Łukasiewicz's three-valued logic," Bulletin of the Section of Logic, vol. 4, pp. 87-90, 1975.

[39] —_ "The Fregean Axiom and Polish mathematical logic in the 1920s," Studia Logica, vol. 36, pp. 377-380, 1977.

[40] H. Wansing and Y. Shramko, "Suszko's Thesis, inferential manyvaluedness, and the notion of a logical system," Studia Logica, vol. 88, no. 3, pp. 405-429, 2008.

[41] R. Wójcicki, Theory of Logical Calculi. Dordrecht: Kluwer, 1988. 